\title{
Radiotherapy Intensified by Increase of Fraction Size Using Three-Dimensional Conformal Radiotherapy in Bladder Preservation Therapy
}

\author{
Kiyoshi Ohara1 $^{*}$, Katsuhiko Oyama1, Akiyoshi Suzuki', Keizo Kawano², Shinji Morimoto ${ }^{2}$, \\ Ayako Okawa ${ }^{1}$ \\ ${ }^{1}$ Department of Radiation Oncology, Tsuchiura Kyodo General Hospital, Tsuchiura City, Japan \\ ${ }^{2}$ Department of Urology, Tsuchiura Kyodo General Hospital, Tsuchiura City, Japan \\ Email: ${ }^{*}$ ki-ohara@tkgh.jp
}

Received 3 September 2015; accepted 20 October 2015; published 23 October 2015

Copyright (C) 2015 by authors and Scientific Research Publishing Inc.

This work is licensed under the Creative Commons Attribution International License (CC BY).

http://creativecommons.org/licenses/by/4.0/

(c) () Open Access

\begin{abstract}
Purpose: The impact of radiotherapy on bladder cancers heightened by increase of fraction size using three-dimensional conformal radiotherapy (3DCRT) was verified with biologically effective dose (BED, Gy10). Method: Twenty-five patients who were considered unsuitable for total cystectomy underwent 3DCRT between 2009 and 2014. When a gross tumor volume (GTV) did not neighbor guts and the patient was cooperative to radiotherapy (radical), the GTV was treated with a fraction size of $\geq 3.0 \mathrm{~Gy}$, while a clinical target volume (CTV) of a whole bladder and lymph nodes at small pelvis were treated with a conventional fraction size of $2.0 \mathrm{~Gy}$. When a GTV neighbored guts (palliative), only a CTV was treated with an increased fraction size of $2.5 \mathrm{~Gy}$. Results: Compared to conventional two-dimensional radiotherapy, BED increased to 62.3 - 77.0 Gy10 (radical) or to 56.1 - $68.0 \mathrm{Gy10}$ (palliative) but the treatment period shortened to 4 - 5 weeks. Acute adverse events ( $\leq 3$ months), determined by common terminology criteria for adverse events, were Grade 2 at most, but late adverse events were not observed. Only one patient $(6 \%)$ lost the bladder. Conclusions: Patients who receive bladder preservation therapy appear good candidates to intensify impact of radiotherapy with 3DCRT.
\end{abstract}

\section{Keywords}

Intensive Radiotherapy, Hypofractionation, Parallel Organ

\footnotetext{
${ }^{*}$ Corresponding author.
}

How to cite this paper: Ohara, K., Oyama, K., Suzuki, A., Kawano, K., Morimoto, S. and Okawa, A. (2015) Radiotherapy Intensified by Increase of Fraction Size Using Three-Dimensional Conformal Radiotherapy in Bladder Preservation Therapy. Journal of Cancer Therapy, 6, 993-999. http://dx.doi.org/10.4236/jct.2015.611107 


\section{Introduction}

In the two-dimensional radiotherapy (2DRT) era, fraction size has been traditionally 1.8 - 2.0 Gy with 5 fractions (Fr) per week, which is called conventional radiotherapy (RT). With 2DRT, the total dose was approximately 60 - $70 \mathrm{~Gy}$, and the treatment period prolonged to 6 - 8 weeks, even in radical intent RT. In addition, normal tissues were usually irradiated largely, which concerned adverse events deeply. Therefore, to heighten the tumor control without heightening the adverse events, change of RT patterns, such as multiple daily fractionations, concurrent uses of chemotherapy, and introduction of particle RT, was considered. But it was not so effective in the last century.

Meanwhile, three-dimensional conformal radiotherapy (3DCRT) can heighten conformity much more than 2DRT. That is, the treatment targets are clearly visible with the progress of diagnostic imaging, and the targets can be irradiated three-dimensionally with the progress of RT techniques. However, most radiation oncologists do not increase fraction size to heighten tumor control, and use conventional fractionation to reduce adverse events. We have carefully selected RT patients with increase of fraction size using 3DCRT techniques. At that time, Koukourakis M.I. et al. [1] treated muscle invasive bladder cancer (MIBC) patients with concomitant boost; so-called hypofractionated accelerated RT, and studied cytoprotection of Amifostine. They treated patients with an increased fraction size of 2.7 Gy to the pelvis, a clinical target volume (CTV), during a short treatment period of 19 days. Furthermore, a boost dose of 0.7 Gy per fraction was given to the bladder concomitantly, i.e., 3.4 Gy per fraction to the bladder. They concluded that increased fraction size using cytoprotection caused higher local control and lower toxicity compared to conventional RT. However, the boost doses were not delivered to the gross tumor volume (GTV) but to the whole bladder. In order to preserve the bladder, it may be more suitable to deliver the boost dose GTV only. The purpose of this study was to estimate the impact of an increased fraction size to GTV on the local tumor control by calculating biologically effective dose (BED, Gy10) [2] [3].

\section{Patients and Method}

\subsection{Patient Characteristics}

The study comprised of 25 radiotherapy patients who refused to be totally cystectomized between July 2009 and March 2014. Of these 25 patients, 9 patients wished for bladder preservation, whereas the remaining 16 patients accepted the preservation therapy because curative total cystectomy was considered difficult to apply. Two patients showed hydronephrosis, and another patient showed cN1 lesion. RT intent was divided into radical ( $\mathrm{n}=18$ ) and palliative $(n=7)$. The patient characteristics are shown in Table 1 . The patient ages at RT ranged 60 - 91 years old (average 79): 14 patients were 80 years old or more (56\%). Eleven patients were female and 14 were male. The performance status with European Cooperative Oncology Group [ECOG] was 0 - 1. However, it was 2 when considering aging. Patient cancers included MIBC $(n=21,84 \%)$, superficial bladder cancer $(n=1)$, and ureteral cancer recurred after surgery with bladder preservation $(n=3)$. Histologic tumor grade was 3 in 20 of 22 patients (88\%), who underwent trans-urethral resection of bladder tumor (TURBT) 2 - 3 months before RT.

\subsection{Treatments}

We used 10 MV X rays for RT. RT treatment unit and treatment planning unit, Clinac iX and Eclipse version 8.6 (both Varian, California, USA), became clinically available in September 2009. The treatment unit served to precisely deliver beams to the patients by moving therapy of a gantry, intensity-modulated RT as well as image guided RT with cone-beam computed tomography (CBCT).

In practice, both the CTV and GTV were contoured by radiation oncologists, normally with $8 \mathrm{~mm}$ of the safety margins. More specifically, the CTV was delineated to cover lymph nodes of the small pelvis and the total bladder. The GTV was delineated on fusion images of the treatment planning CT images with the MRI or CT images before TURBT.

In radical RT, when the patient was cooperative to RT and GTV did not involve guts, GTV was usually located at the bladder base. CTV was treated with a conventional fraction size of 2.0 Gy using a box field technique of 2DRT, and GTV was treated with fraction sizes of $\geq 3.0$ Gy using moving techniques of 3DCRT.

GTV was treated by one of the following three methods: 1$)$ sequential boost $(n=4)$; 2) GTV only $(n=6)$ and 3) concurrent boost $(n=8)$. As to the sequential method, after CTV was treated with $45.0 \mathrm{~Gy} / 15 \mathrm{Fr}(\mathrm{n}=1)$ or 46.0 Gy/23 Fr (n=3), GTV was treated over 5 Fr with fraction size of 4.0 or 5.0 Gy, i.e., 70.0 Gy/20 Fr or 
Table 1. Patient characteristics.

\begin{tabular}{|c|c|c|c|}
\hline Treatment Intent & Radical & Palliative & Total \\
\hline & 18 & 7 & 25 \\
\hline Age (Average): Years & \multicolumn{2}{|c|}{60 - 91 (79) } & \\
\hline Male:Female & \multicolumn{2}{|c|}{$14: 11$} & \\
\hline Performance Status (ECOG) & \multicolumn{2}{|c|}{0 - 1 (2: by Aging) } & \\
\hline \multicolumn{4}{|l|}{ Disease } \\
\hline MIBC & 15 & 6 & 21 \\
\hline Superficial BC & 0 & 1 & 1 \\
\hline Recurrent Ureteral Cancer & 3 & 0 & 3 \\
\hline \multicolumn{4}{|l|}{ TURBT before Radiotherapy } \\
\hline+ & 16 & 6 & 22 \\
\hline- & 2 & 1 & 3 \\
\hline \multicolumn{4}{|l|}{ Histologic Grade (TURBT+) } \\
\hline Grade 3 & 15 & 5 & 20 \\
\hline Grade 2 & 1 & 1 & 2 \\
\hline \multicolumn{4}{|l|}{ Concurrent Chemotherapy (CDDP) } \\
\hline+ & 11 & 3 & 14 \\
\hline- & 7 & 4 & 11 \\
\hline \multicolumn{4}{|l|}{ Radiothapy Volume } \\
\hline GTV + CTV & 12 & 2 & 14 \\
\hline GTV Alone & 6 & 1 & 7 \\
\hline CTV Alone & 0 & 4 & 4 \\
\hline
\end{tabular}

ECOG = European Cooperative Oncology Group; $\mathrm{MIBC}=$ muscle invasive bladder cancer; TURBT = trans-urethral resectionof bladder tumor; $\mathrm{CDDP}=$ cis-diamminedichloro platinum (II); GTV = gross tumor volume, CTV = clinical target volume.

66.0 Gy/28 Fr - 71.0 Gy/28 Fr, respectively. As to the GTV only method, GTV was treated with a fraction size of $2.5 \mathrm{~Gy}(\mathrm{n}=1)$ or $3.0 \mathrm{~Gy}(\mathrm{n}=5)$, i.e., $65.0 \mathrm{~Gy} / 26 \mathrm{Fr}$ or $60.0 \mathrm{~Gy} / 2 \mathrm{Fr}-72.0 \mathrm{~Gy} / 24 \mathrm{Fr}$, respectively. As to the concurrent boost method, GTV was simultaneously boosted with a fraction size of 3.0 Gy, i.e., CTV 46.0 Gy and GTV 69.0 Gy over 23 Fr. Patients instructed to urinate 60 minutes before RT, so that the bladder could be expanded.

In palliative RT, when the patient was not cooperative to RT or GTV neighbored guts, GTV was usually located at the bladder dome. Only CTV was treated with 2DRT with an increased fraction size of $2.5 \mathrm{~Gy}(\mathrm{n}=5)$ to shorten the treatment period: $50.0 \mathrm{~Gy} / 20 \mathrm{Fr}$ or $60.0 \mathrm{~Gy} / 24 \mathrm{Fr}$.

Fourteen patients (56\%) received concurrent chemo-radiotherapy (CCRT). In CCRT, cisplatinum (CDDP) was used: $10 \mathrm{mg}$ per body per day for the first 5 consecutive RT days and also for the last 5 consecutive RT days ( $\mathrm{n}=11,44 \%$ ). The CDDP dose was adjusted according to hematologic toxicities or to aging. Eight patients received intra-vesical chemotherapy, whereas four patients did not receive any chemotherapy.

\subsection{BED Calculation}

The BED based on the linear-quadratic model was used [3] to calculate tumor impacts on GTV in radical RT and on CTV in palliative RT. 
The BED (Gy10) was calculated as follows:

$$
\mathrm{BED}=n \times d(1+d / \alpha / \beta)-0.693 \times t / \alpha \times \mathrm{Tp}
$$

where, $n$ is the number of fractions, $d$ is each fraction size (Gy), and $t$ is the treatment period (days). We assumed that $\alpha / \beta$ for bladder tumor was 10 (Gy), $\alpha$ was 0.3 , and Tp (potential doubling time) was 5 (days).

\subsection{Adverse Events Evaluation}

Adverse events were evaluated with CTACE version 4.0 [4], and the concerned toxicities were as follows: hematologic toxicities of blood and lymphatic system disorders, gastrointestinal toxicities of intestinal disorders, genitourinary toxicities of renal and urinary disorders, and other toxicities of general disorders. Adverse events occurring during 3 months after the initiation of RT were graded as early adverse events. Late adverse events were defined as those occurring more than 3 months after the RT.

\subsection{Follow-Up Examinations}

Follow-up examinations were performed every 3 - 4 months after RT. The examinations included physical examinations, laboratory tests, urine cytology, cystoscopy, and diagnostic imaging of CT or MRI. When the recurrence was suspected, TURBT underwent when feasible.

\subsection{Statistical Analysis}

StatView 5.0 (SAS Institute Inc., North Carolina, USA) was used for all statistical analyses. Data were calculated from the initiation of RT. Kaplan-Meyer method was used for determination of rates.

\section{Results}

The patients or their family accepted the treatments with the written agreements. Treatment results are shown in Table 2. BED was calculated according to Equation (1) to be 62.3 - 77.0 Gy10 for radical RT patients and 56.1 68.0 Gy10 for palliative RT patients. Even in palliative RT, BED was higher compared to 43.0 Gy10 or 53.5 Gy10, i.e., $50.0 \mathrm{~Gy} / 25 \mathrm{Fr}$ or $60.0 \mathrm{~Gy} / 30 \mathrm{Fr}$ with conventional fractionation. The treatment periods were shortened to 4 - 5 weeks. RT interruptions were not encountered. Acute adverse events were Grade 2 or less: Grade 0 $(n=10)$, Grade $1(n=7)$, and Grade $2(n=8)$. No patients with Grade 3 or more of adverse events were observed. The most frequent Grade 2 adverse events were hematologic toxicities. Other Grade 2 toxicities were urinary toxicities of cystitis $(n=2)$ and intestinal toxicities of diarrhea $(n=1)$. We did not observe late adverse events during the follow-up periods. Follow-up TURBT was performed for 10 patients (40\%): 5 patients showed no viable tumor cells, whilst the remaining 5 patients showed superficial carcinoma.

\begin{tabular}{lccc} 
Table 2. Treatment results. & & & \\
\hline \multicolumn{1}{c}{ Treatment Intent } & Radical & Palliative & Total \\
\hline & 18 & 7 & 25 \\
BED (Gy10) & $62.3-77.0$ & $56.1-68.0$ & \\
Treatment Period (Days) & $20-28$ & $17-24$ & \\
Adverse Events & & & \\
$\quad$ Grade 0 & 4 & 6 & 10 \\
$\quad$ Grade 1 & 7 & 0 & 7 \\
$\quad$ Grade 2 & 7 & 1 & 0 \\
$\quad$ Grade 3 - 5 & 0 & 0 & 10 \\
Follow-Up TURBT & 9 & 1 & 8 \\
\hline
\end{tabular}

BED = biologically effective dose; TURBT = trans-ureteral resection of bladder tumor. 
The follow-up period ranged 4.4 - 66.0 months (median 25.1) at 31 March 2015. We were unable to follow up 8 patients (32\%) by aging, but all of 8 patients were alive with no evidence of recurrence. As follow-up examinations, cystoscopy $(n=18,72 \%)$ and diagnostic imaging $(n=23,92 \%)$ were performed.

Five patients were died: 4 were MIBC patients and one was a superficial bladder cancer patient (Figure 1). Three of them received radical RT: one died of lung cancer with the bladder tumor controlled, another died of systemic metastases with local recurrence, and the remaining died of age (88 years) with local recurrence (cT1). The remaining two received palliative RT: one showed lymph-node metastasis with local disease control (cN1), and another with superficial bladder cancers eventually received total cystectomy, that showed sarcoma-changes. Tumor recurrence was identified in 10 patients, who were regarded as dead in the analysis of progression free survival. Rates of 3-year overall survival and 3-year progression free survival were $64 \%$ and $42 \%$, respectively. Bladder preservation rate was $94 \%$.

\section{Discussion}

In the 2DRT era, radiation tolerance was determined mainly by normal tissue [5]. In the 3DCRT era, however, it would be determined mainly by tumor tissue itself. Bladder tumor tissue will cause ulceration or necrosis resulting in perforation of the bladder. Radiation tolerance with 3DCRT was not fully understood [6], and neither was hypofractionation [7] [8]. Our patients tolerated the high BED RT in terms of acute adverse events. However, major concerns of radiation oncologists will be late adverse events.

Techniques of 3DCRT have been successfully used in stereotactic body radiotherapy (SBRT) for non-small cell lung cancers or liver tumors [9] [10]. Lung and liver have been considered as parallel organs in radiation tolerance of normal tissues in 2DRT era. These tumors were treated by very high doses to GTV with very small volumes to normal tissues. For example, in SBRT of non-small cell lung cancer of cT1-2N0M0, fraction sizes are as high as 10.0 or $12.0 \mathrm{~Gy}$, with a total dose of 48.0 or $50.0 \mathrm{~Gy}$ over 4 - $5 \mathrm{Fr}$ [9]. In this treatment, the tumor impact with BED is calculated to be $97 \mathrm{~Gy} 10$ and is equivalent to110.0 Gy/55 Fr over 11 weeks with conventional fractionation.

The gold standard of care for MIBC is total cystectomy [11]. However, total cystectomy reduces the patients' quality of life substantially. The bladder is known through 2DRT treatments of uterine cervical cancer to be an organ that could tolerate high doses [12]-[15]. It could tolerate even in association with high-dose-rate brachytherapy [12]. Therefore, the patients with bladder preservation therapy were considered safely treated with high dose RT when use techniques of 3DCRT.

We initially referred to concomitant boost of hypofractionated accelerated $\mathrm{RT}^{1}$ and later to their clinical results [16]. Their fraction size of 3.4 Gy, 51.0 Gy/19 Fr with 2DRT, is calculated to be 59.6 Gy10 with the BED, which corresponds to $68.0 \mathrm{~Gy} / 34$ Fr over 46 days. Their dose is almost equal to our palliative RT of 49.1 - 58.8

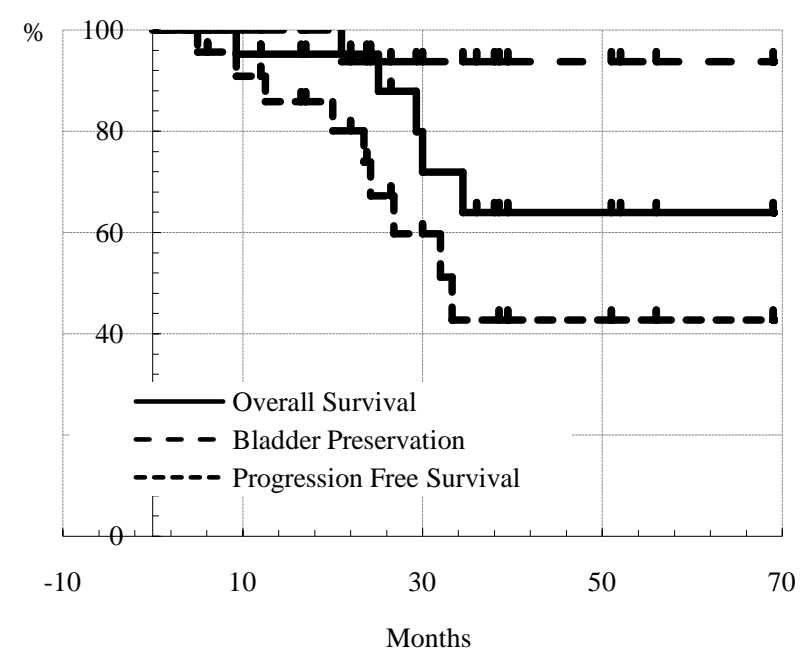

Figure 1. Data of patients received bladder preservation radiotherapy $(n=25)$. Over all survival rate, bladder preservation rate, and progression free survival rate are shown. 
Gy10. Our data of 3-year overall survival of 64\% may not be very high compared to the literature [17]-[21]. However, treatment periods were shortened to 4 - 5 weeks instead of 6 - 9 weeks with conventional RT. Shortening of treatment periods without adverse events are beneficial particularly to elderly patients who have to visit hospitals for RT. Besides, bladder was preserved in $94 \%$ of patients.

The causes of death of the MIBC patients were mostly local recurrences. The strongest treatment of nonoperative is considered to be trimodality therapy [17] [18]. It is important in this setting to intensify RT, however, adequate delineation of the GTV and reproducibility in each RT session are essential. Currently, we treat patients with a concurrent boost method by delivering a total dose of $72.0 \mathrm{~Gy} / 24 \mathrm{Fr}$ to GTV, where CBCT [22] is routinely used to verify the reproducibility. In these cases, BED heightens 78.8 Gy10 to GTV, which is calculated to be equivalent to $90.0 \mathrm{~Gy} / 45$ Fr over 64 days with conventional RT. Limitations of the study include the small number of patients and the shortness of their follow up periods particularly to see late adverse events.

\section{Conclusion}

The increase of fraction size using 3DCRT tolerated well at least acute adverse events in the bladder preservation RT. Much more patients are needed to verify the safety of 3DCRT concretely including late adverse events. Besides, since the current model of BED was developed in the 2DRT era, we must use the model carefully and improve it suitable to 3DCRT.

\section{Conflicts of Interest}

We affirm that we do not have any financial or other relations, conflicts of interest. We also affirm that this manuscript is original, has not been published previously, is not under consideration by another journal, and will not be submitted elsewhere until we have received your final decision.

\section{References}

[1] Koukourakis, M.I., Tsolos, C. and Tuoloupidis, S. (2007) Radical Hypofractionated Accelerated Radiotherapy with Cytoprotection for Invasive Bladder Cancer. Urology, 69, 245-250. http://dx.doi.org/10.1016/j.urology.2006.09.064

[2] Astrahan, M. (2008) Some Implications of Linear-Quadratic-Linear Radiation Dose-Response with Regard to Hypofractionation. Medical Physics, 35, 4161-4172. http://dx.doi.org/10.1118/1.2969065

[3] Fowler, J.F. (2010) 21 Years of Biologically Effective Dose. British Journal of Radiology, 83, 554-568.

[4] National Cancer Institute. Cancer Therapy Evaluation Program. Common Terminology Criteria for Adverse Events (CTCAE) Version 4. http://ctep.cancer.gov/protocolDevelopment/adverse effects.htm

[5] Emami, B., Lyman, J., Brown, A., et al. (1991) Tolerance of Normal Tissue to Therapeutic Irradiation. International Journal of Radiation Oncology*Biology*Physics, 21, 109-122. http://dx.doi.org/10.1016/0360-3016(91)90171-Y

[6] Marks, L.B., Yorke, E.D., Jackson, A., et al. (2010) Use of Normal Tissue Complication Models in the Clinic. International Journal of Radiation Oncology*Biology*Physics, 76, S10-S19. http://dx.doi.org/10.1016/j.ijrobp.2009.07.1754

[7] Timmeman, R.D. (2008) An Overview of Hypofractionation and Introduction to This Issue of Seminars in Radiation Oncology. Seminars in Radiation Oncology, 18, 215-222.

[8] Hamilton, J., Higgins, G. and Bernhard, E.J. (2009) Conventional Radiotherapy or Hypofractionation? A Study of Molecular Changes Resulting from Different Radiation Fraction Schemes. Cancer Biology \& Therapy, 8, 774-776. http://dx.doi.org/10.4161/cbt.8.9.8341

[9] Matsuo, Y., Chen, F., Hamaji, M., et al. (2014) Comparison of Long-Term Survival Outcomes between Stereotactic Body Radiotherapy and Sublobar Resection for Stage I Non-Small-Cell Lung Cancer in Patients at High Risk for Lobectomy: A Propensity Score Matching Analysis. European Journal of Cancer, 50, 2932-2938. http://dx.doi.org/10.1016/j.ejca.2014.09.006

[10] Yamashita, H., Onishi, H., Murakami, N., et al. (2015) Survival Outcomes after Stereotactic Body Radiotherapy for 79 Japanese Patients with Hepatocellular Carcinoma. Journal of Radiation Research, 53, 561-567. http://dx.doi.org/10.1093/jrr/rru130

[11] National Cancer Institute. Bladder Cancer Treatment (PDQ). 31 March 2015, Date Last Accessed. http://www.cancer.gov/cancertopics/pdq/treatment/bladder/HealthProfessional

[12] Nag, S., Erickson, B., Thomadsen, B., Orton, C., Demanes, J.D. and Petereit, D. (2000) The American Brachytherapy Society Recommendations for High-Dose-Rate Brachytherapy for Carcinoma of the Cervix. International Journal of Radiation Oncology, Biology, Physics, 48, 201-211. http://dx.doi.org/10.1016/S0360-3016(00)00497-1 
[13] Sundar, S., Symonds, P. and Deehan, C. (2003) Tolerance of Pelvic Organs to Radiation Treatment for Carcinoma of Cervix. Clinical Oncology, 15, 240-247. http://dx.doi.org/10.1016/S0936-6555(02)00455-7

[14] National Cancer Institute. Cervical Cancer Treatment (PDQ). 31 March 2015, Date Last Accessed. http://www.cancer.gov/cancer_information/

[15] Viswanathan, A.N., Yorke, E.D., Marks, L.B., Eifel, P.J. and Shipley, W.U. (2010) Radiation Dose-Volume Effects of the Urinary Bladder. International Journal of Radiation Oncology, Biology, Physics, 76, S116-S122. http://dx.doi.org/10.1016/j.ijrobp.2009.02.090

[16] Panteliadou, M., Giatromanolaki, A., Touloupidis, S., Destouni, E., Tsoutsou, P.G., Pantelis, P., et al. (2012) Treatment of Invasive Bladder Cancer with Conformal Hopofractionated Accelerated Radiotherapy and Amifostine (HypoARC). Urologic Oncology, 30, 813-820. http://dx.doi.org/10.1016/j.urolonc.2010.09.001

[17] Mitin, T., Shipley, W.U., Efstathiuo, J.A., Heney, N.M., Kaufman, D.S., Lee, R.J. and Zietman, A.L. (2013) Trimodality Therapy for Bladder Conservation in Treatment of Invasive Bladder Cancer. Current Urology Reports, 14, 109-115. http://dx.doi.org/10.1007/s11934-012-0301-x

[18] Ploussard, G., Doneshmand, S., Efstathiou, J., Herr, H.W., James, N.D., Rödel, C.M., et al. (2014) Critical Analysis of Bladder Sparing with Trimodal Therapy in Muscle-Invasive Bladder Cancer: A Systematic Review. European Urology, 66, 120-137. http://dx.doi.org/10.1016/j.eururo.2014.02.038

[19] Smith, Z.L., Christodouleas, J.P., Keefe, S.M., Malkowicz, S.B. and Guzzo, T.J. (2013) Bladder Preservation in the Treatment of Muscle-Invasive Bladder Cancer (MIBC): A Review of the Literature and a Practical Approach to Therapy. BJU International, 112, 13-25. http://dx.doi.org/10.1111/j.1464-410X.2012.11762.x

[20] Foroudi, F., Pham, D., Rolfo, A., Bressel, M., Tang, C.I., Tan, A., et al. (2014) The Outcome of a Multi-Centre Feasibility Study of Online Adaptive Radiotherapy for Muscle-Invasive Bladder Cancer TROG 10.01 BOLART. Radiotherapy and Oncology, 111, 306-320. http://dx.doi.org/10.1016/j.radonc.2014.02.015

[21] Turgeon, G.A., Souhami, L., Cury, F.L., Faria, S.L., Duclos, M., Sturgeon, J. and Kassouf, W. (2014) Hypofractionated Intensity Modulated Radiation Therapy in Combined Modality Treatment for Bladder Preservation in Elderly Patients with Invasive Bladder Cancer. International Journal of Radiation Oncology, Biology, Physics, 88, 326-331. http://dx.doi.org/10.1016/j.ijrobp.2013.11.005

[22] Lalondrelle, S., Huddart, R., Warren-Oseni, K., Hansen, V.N., McNair, H., Thomas, K., et al. (2011) Adaptive-Predictive Organ Localization Using Cone-Beam Computed Tomography for Improved Accuracy in External Beam Radiotherapy for Bladder Cancer. International Journal of Radiation Oncology, Biology, Physics, 79, 705-712. http://dx.doi.org/10.1016/j.ijrobp.2009.12.003 